\title{
Characterization of lentil (Lens culinaris M.) germplasm through phenotypic marker
}

\author{
R. Gupta, S. N. Begum ${ }^{1}$, M. M. Islam ${ }^{2}$ and M. S. Alam \\ Department of Genetics and Plant Breeding, Bangladesh Agricultural University, Mymensingh-2202, Bangladesh, \\ ${ }^{1}$ Plant Breeding Division and and ${ }^{2}$ Biotechnology Division, Bangladesh Institute of Nuclear Agriculture, Mymensingh- \\ 2200, Bangladesh, E-mail: rigyan2008@gmail.com
}

\begin{abstract}
An experiment was carried out to evaluate the performance of 22 lentil (Lens culinaris M.) genotypes for their yield and yield contributing characters at phenotypic level, relationship and diversity among the individuals and characterize the germplasm through phenotypic markers. ANOVA table revealed that significant variation was found among the genotypes for the characters plant height, days to maturity, number of primary branches plant ${ }^{-1}$, number of secondary branches plant ${ }^{-1}$, height of the lowest pod and 100-seed weight. Yield plant ${ }^{-1}$ was positively correlated with pods plant ${ }^{-1}$, seeds pod $^{-1}$, seeds plant ${ }^{-1}$ and 100-seed weight. Considering yield plant ${ }^{-1}$ LM-123-7 was the best followed by LM-132-7, ICARDA-2348 and LM-13-1. Days to flowering, days of maturity and 100-seed weight showed high heritability. Path analysis revealed that pods plant ${ }^{-1}$ and 100 -seed weight had positive direct effects on yield plant $^{-1}$. The genotypes were grouped into five clusters based on Euclidean distance following Ward's method and the highest intra-cluster distance was found in cluster IV and inter-cluster distance between the cluster IV and cluster V. Study of selection indices through discriminate functions observed that LM-123-7 ranked as the best followed by LM-132-7, LM-21-6, LM-24-3 and LM-14-2 and suggests that the highest scoring genotypes might be recommended for farmer's cultivation for better yield.
\end{abstract}

Keywords: Lentil genotypes, Coefficients of correlation, Path analysis, Clustering, Selection index

\section{Introduction}

Lentil (Lens culinaris ssp. culinaris) is a diploid $(2 n=2 x=14)$, autogamous species and is one of the oldest crops in the world, which originated in the Near East (Zohary, 1972). The cultivated lentil belongs to the family Leguminosae, sub-family Papilionaceae. The genus Lens comprises seven taxa within four species including the cultivated type, Lens culinaris spp. culinaris (Ferguson and Erskine, 2001). Cultivated lentil includes two varietal types: small-seeded microsperma and large-seeded macrosperma. The major lentil producing regions are Asia (58 percent of the area) and the West Asia-North Africa region (37 percent of the acreage of developing countries). In 2010, the total area used in the whole world was 4189502 ha where the total production was 4585439 tons. In Bangladesh the land area used for lentil cultivation was 77287 ha and the production of lentil was about 71100 tons (FAOSTAT, 2010). The largest and most representative collection of lentil landraces is maintained by the International Center for Agricultural Research in the Dry Areas (ICARDA) (3351 accessions), and large collections are also held in the USA (2807 accessions), Russia (2857 accessions), Australia (2847 accessions) and Turkey (1435 accessions); (Ford et al., 2007). Several of the lentil landraces that have exhibited significantly superior root and shoot traits and yield were considered to be valuable germplasms for breeding a drought-tolerant cultivar (Sarker et al., 2005). In Bangladesh, the lentil landraces must be characterized for use of the future lentil breeding program. So, this experiment is conducted to evaluate the performance of 22 lentil (Lens culinaris M.) genotypes by phenotypic markers and to determine the relationship and diversity among the individuals.

\section{Materials and Methods}

The experiment was conducted at the experimental farm of Bangladesh Institute of Nuclear Agriculture (BINA), Mymensingh, during November 2011 to March 2012. Geographically the experimental area is located at $24^{0} 75 \mathrm{~N}$ latitude and $90^{\circ} 5 \mathrm{E}$ longitudes at the elevation of $18 \mathrm{~m}$ above the sea level. The field was medium high land. The soil of the experimental site belonging under the agro-ecological zone of Old Brahmaputra Floodplain (AEZ-9). The experimental field area was under sub-tropical climates characterized by heavy rainfall during the month of April to September and scanty rainfall during October 
to March. The seeds of sixteen advanced lentil mutants ( i.e. LM-123-7, LM-132-7, LM-28-2, LM-14-2, LM-21-6, LM-24-3, LM-37-8, LM-48-1, LM-99-4, LM-95-3, LM-13-1, LM-156-1, LM-185-2, LM-20-3, LM67-5 and LM-75-4) and six ICARDA lines (i.e. ICARDA 2348, ICARDA 38211, ICARDA 23136, ICARDA 23121, ICARDA 23105 and ICARDA 23128) were collected from Plant Breeding Division, Bangladesh Institute of Nuclear Agriculture, Mymensingh. The experiment was laid out in a Randomized Complete Block Design (RCBD) with five replications. The experimental field was divided into five blocks each representing one replication. Each block was then sub-divided in to twenty two plots. The sixteen lentil mutants and six ICARDA lines was placed in each plot. The size of the unit plot was $2 \mathrm{~m} \times 1.2 \mathrm{~m}$. Row to row and plant to plant distances were $30 \mathrm{~cm}$ and $5 \mathrm{~cm}$, respectively in each plot. The growth, yield and yield-contributing characters plant height $(\mathrm{cm})$, number of primary branches plant ${ }^{-1}$, number of secondary branches plant $^{-1}$, days to flowering, days to maturity, height of the lowest pod $(\mathrm{cm})$, number of pods plant ${ }^{-}$ 1 , seeds pod $^{-1}$, seeds plant ${ }^{-1}, 100$-seed weight and yield plant $^{-1}$ were recorded for phenotypic characterization. The statistical analysis was done by plant breeding statistical program (PLBSTAT, Version 2N, Utz, 2007). Estimation of heritability, estimation of correlation coefficients, estimation of path co-efficient, analysis of genetic divergence, euclidean distance, clustering and estimation of selection index were done as per objectives.

\section{Results and Discussion}

The performance of different lentil genotypes for grain yield and different yield contributing characters were evaluated and there were significant variations among the genotypes for the characters plant height, days to maturity, number of primary branches plant ${ }^{-1}$, number of secondary branches plant ${ }^{-1}$, height of the lowest pod and 100-seed weight (Table 1).

Table 1. Analysis of variance for plant characters of 22 lentil genotypes

\begin{tabular}{|c|c|c|c|c|c|c|c|c|c|c|c|c|}
\hline Items & d. f. & $\begin{array}{l}\text { Plant } \\
\text { height } \\
\text { (cm) }\end{array}$ & $\begin{array}{l}\text { Days to } \\
\text { flowering }\end{array}$ & $\begin{array}{l}\text { Days of } \\
\text { maturity }\end{array}$ & $\begin{array}{c}\text { No. of primary } \\
\text { branches } \\
\text { plant }^{-1}\end{array}$ & $\begin{array}{c}\text { No. of } \\
\text { secondary } \\
\text { branches plant }{ }^{-1}\end{array}$ & $\begin{array}{l}\text { Height of } \\
\text { the lowest } \\
\operatorname{pod}(\mathrm{cm})\end{array}$ & $\begin{array}{l}\text { No. of pod } \\
\text { plant }^{-1}\end{array}$ & $\begin{array}{l}\text { No. of } \\
\text { seeds } \\
\text { pod-1 }\end{array}$ & $\begin{array}{l}\text { No. of } \\
\text { seeds } \\
\text { plant-1 }\end{array}$ & $\begin{array}{l}100 \text { seed } \\
\text { weight }(\mathrm{g})\end{array}$ & $\begin{array}{l}\text { Yield } \\
\text { plant }^{-1} \\
\text { (g) }\end{array}$ \\
\hline Genotypes & 21 & $63.72^{\star *}$ & $210.69^{\star \star}$ & $34.95^{\star \star}$ & $6.50^{* *}$ & $41.31^{*}$ & $26.49^{\star *}$ & 9636 & 0.07 & 24642 & $0.59 * *$ & 12.96 \\
\hline Replication & 4 & 47.57 & 15.15 & 9.58 & 2.47 & $61.24^{*}$ & 7.70 & 3501 & 0.07 & 20626 & 0.03 & 10.20 \\
\hline Error & 84 & 23.10 & 19.14 & 7.34 & 2.73 & 24.51 & 7.56 & 7143 & 0.04 & 18287 & 0.10 & 9.03 \\
\hline
\end{tabular}

${ }^{*}$ and ${ }^{* *}$ indicates significant at 0.05 and 0.01 probability, respectively

In Table 2 it was found that the genotype ICARDA 23128 was the tallest plant and the genotype LM-28-2 was the shorter one. LM-24-3 was the earlier flowering genotype and the genotypes, LM-185-2, ICARDA23105, LM-67-5 took the highest days to maturity (110 days) and the LM-37-8 was an early maturing one (102 days) this result was in full agreement with Dutta (2001). The genotype ICARDA-23136 had highest primary branches and the genotype ICARDA-23128 had highest secondary branches. The genotypes, ICARDA-38211 and ICARDA-23105 were maintained maximum height of the lowest pod then other genotypes. The highest number of pods plant ${ }^{-1}$ was recorded in ICARDA- 2348 and the lowest was recorded in ICARDA-23121. The highest number of seeds pod $^{-1}$ was recorded in LM-13-1 and lowest number was found in ICARDA-23136. The lowest seeds plant $^{-1}$ was found in ICARDA-23136 and the highest was found in ICARDA- 2348 followed by ICARDA-23128. The highest 100-seed weight was recorded in LM-24-3 and the lowest was found in LM-37-8. The highest yield plant ${ }^{-1}$ was recorded in LM123-7 and the lowest in ICARDA-23136. Among the 11 characters, 50\% flowering, days of maturity and 100 -Seed weight were high heritable and plant height, number of primary branches plant ${ }^{-1}$, number of secondary branches plant ${ }^{-1}$, height of the lowest pod and number of seeds pod $^{-1}$ were medium heritable and number of pod plant ${ }^{-1}$, number of seeds plant ${ }^{-1}$ and yield plant ${ }^{-1}$ were low heritable (Table 3 ). 
Gupta et al.

Table 2. Mean performance of 22 genotypes based on different morphological traits related to yield

\begin{tabular}{|c|c|c|c|c|c|c|}
\hline Genotypes & $\begin{array}{c}\text { Plant height } \\
(\mathrm{cm})\end{array}$ & $\begin{array}{l}\text { Days to } \\
\text { flowering }\end{array}$ & $\begin{array}{l}\text { Days of } \\
\text { maturity }\end{array}$ & $\begin{array}{l}\text { No. of primary } \\
\text { branches plant }^{-1}\end{array}$ & $\begin{array}{l}\text { No. of secondary } \\
\text { branches plant }^{-1}\end{array}$ & $\begin{array}{c}\text { Height of the } \\
\text { lowest pod }(\mathrm{cm})\end{array}$ \\
\hline LM-123-7 & $27.10 \mathrm{c}-\mathrm{f}$ & $54.40 \mathrm{f}-\mathrm{i}$ & 107 a-f & $4.20 \mathrm{bc}$ & $12.80 \mathrm{a}-\mathrm{c}$ & $5.80 \mathrm{~b}$ \\
\hline LM-132-7 & $31.60 \mathrm{a}-\mathrm{e}$ & $51.80 \mathrm{hi}$ & $106 \mathrm{~b}-\mathrm{f}$ & $3.80 \mathrm{bc}$ & $10.80 \mathrm{bc}$ & $6.68 \mathrm{~b}$ \\
\hline LM-28-2 & $23.60 \mathrm{f}$ & $56.00 \mathrm{~d}-\mathrm{i}$ & $106 \mathrm{~b}-\mathrm{f}$ & $3.80 \mathrm{bc}$ & $8.00 \mathrm{c}$ & $5.80 \mathrm{~b}$ \\
\hline LM-14-2 & $28.20 \mathrm{~b}-\mathrm{f}$ & $51.40 \mathrm{i}$ & $106 \mathrm{~b}-\mathrm{f}$ & $3.80 \mathrm{bc}$ & $7.80 \mathrm{c}$ & $5.60 \mathrm{~b}$ \\
\hline LM-21-6 & $25.00 \mathrm{ef}$ & $53.20 \mathrm{~g}-\mathrm{i}$ & $107 a-f$ & $3.60 \mathrm{bc}$ & $11.40 \mathrm{bc}$ & $6.20 \mathrm{~b}$ \\
\hline LM-24-3 & $26.20 \mathrm{~d}-\mathrm{f}$ & $43.80 \mathrm{j}$ & $102 \mathrm{gh}$ & $4.60 \mathrm{bc}$ & $9.80 \mathrm{bc}$ & $6.00 \mathrm{~b}$ \\
\hline LM-37-8 & $34.70 \mathrm{ab}$ & $51.80 \mathrm{hi}$ & $102 \mathrm{~h}$ & $3.20 \mathrm{c}$ & $9.40 \mathrm{bc}$ & $7.60 \mathrm{~b}$ \\
\hline LM-48-1 & 29.40 a-f & $57.20 \mathrm{~d}-\mathrm{i}$ & $105 d-h$ & $4.40 \mathrm{bc}$ & $10.20 \mathrm{bc}$ & $6.40 \mathrm{~b}$ \\
\hline LM-99-4 & $26.80 \mathrm{c}-\mathrm{f}$ & $56.00 \mathrm{~d}-\mathrm{i}$ & $105 d-h$ & $4.20 \mathrm{bc}$ & $11.40 \mathrm{bc}$ & $7.60 \mathrm{~b}$ \\
\hline LM-95-3 & $24.40 \mathrm{ef}$ & $61.60 \mathrm{~b}-\mathrm{e}$ & $103 \mathrm{f}-\mathrm{h}$ & $3.20 \mathrm{c}$ & $9.00 \mathrm{c}$ & $8.20 \mathrm{~b}$ \\
\hline LM-13-1 & $27.60 \mathrm{~b}-\mathrm{f}$ & $57.80 \mathrm{~d}-\mathrm{i}$ & 104 e-h & $5.60 \mathrm{bc}$ & $13.80 \mathrm{a}-\mathrm{c}$ & $6.20 \mathrm{~b}$ \\
\hline LM-156-1 & $27.80 \mathrm{~b}-\mathrm{f}$ & $60.20 \mathrm{c}-\mathrm{f}$ & $108 \mathrm{a}-\mathrm{d}$ & $5.40 \mathrm{bc}$ & $12.80 \mathrm{a}-\mathrm{c}$ & $6.60 \mathrm{~b}$ \\
\hline LM-185-2 & $26.60 \mathrm{~d}-\mathrm{f}$ & $62.40 \mathrm{~b}-\mathrm{d}$ & $110 \mathrm{a}$ & $5.00 \mathrm{bc}$ & $11.60 \mathrm{bc}$ & $6.44 \mathrm{~b}$ \\
\hline LM-20-3 & 29.60 a-f & $55.80 \mathrm{e}-\mathrm{i}$ & $108 \mathrm{a}-\mathrm{d}$ & $5.40 \mathrm{bc}$ & $11.80 \mathrm{bc}$ & $6.80 \mathrm{~b}$ \\
\hline LM-67-5 & $31.20 \mathrm{a}-\mathrm{e}$ & $58.40 \mathrm{~d}-\mathrm{g}$ & $110 a b$ & $5.80 \mathrm{~b}$ & $11.80 \mathrm{bc}$ & $6.30 \mathrm{~b}$ \\
\hline LM-75-4 & $27.40 \mathrm{~b}-\mathrm{f}$ & $60.80 \mathrm{c}-\mathrm{f}$ & $109 a-c$ & $4.20 \mathrm{bc}$ & $9.00 \mathrm{c}$ & $6.20 \mathrm{~b}$ \\
\hline ICARDA 2348 & $32.80 \mathrm{a}-\mathrm{d}$ & $66.20 \mathrm{a}-\mathrm{c}$ & $109 a-d$ & $5.00 \mathrm{bc}$ & $11.60 \mathrm{bc}$ & $9.10 \mathrm{~b}$ \\
\hline ICARDA 38211 & $34.00 \mathrm{a}-\mathrm{c}$ & $67.40 \mathrm{ab}$ & $110 a b$ & $5.60 \mathrm{bc}$ & $14.40 \mathrm{a}-\mathrm{c}$ & $14.20 \mathrm{a}$ \\
\hline ICARDA 23136 & $29.40 \mathrm{~b}-\mathrm{f}$ & $67.20 \mathrm{ab}$ & $107 \mathrm{a}-\mathrm{e}$ & $8.20 \mathrm{a}$ & $16.80 \mathrm{ab}$ & $9.40 \mathrm{~b}$ \\
\hline ICARDA 23121 & $30.20 a-f$ & $65.80 \mathrm{a}-\mathrm{c}$ & $108 \mathrm{a}-\mathrm{d}$ & $5.40 \mathrm{bc}$ & $14.80 \mathrm{a}-\mathrm{c}$ & $9.40 \mathrm{~b}$ \\
\hline ICARDA 23105 & $34.60 \mathrm{ab}$ & $58.20 \mathrm{~d}-\mathrm{h}$ & $110 \mathrm{ab}$ & $5.00 \mathrm{bc}$ & $13.60 \mathrm{a}-\mathrm{c}$ & $13.20 \mathrm{a}$ \\
\hline ICARDA 23128 & $36.60 a$ & $71.20 \mathrm{a}$ & $108 \mathrm{a}-\mathrm{d}$ & $6.00 \mathrm{~b}$ & $19.80 \mathrm{a}$ & $8.80 \mathrm{~b}$ \\
\hline CV (\%) & 16.40 & 7.47 & 2.54 & 17.52 & 14.51 & 13.66 \\
\hline Maximum & 36.6 & 71.2 & 110 & 8.2 & 19.8 & 14.2 \\
\hline Minimum & 23.6 & 43.8 & 102 & 3.2 & 7.8 & 5.6 \\
\hline Mean & 29.31 & 58.57 & 106.78 & 4.79 & 11.93 & 7.66 \\
\hline LSD (0.05) & 6.04 & 5.50 & 3.41 & 2.08 & 6.23 & 3.46 \\
\hline
\end{tabular}

Table 2. Contd.

\begin{tabular}{|c|c|c|c|c|c|}
\hline Genotypes & No. of pod plant ${ }^{-1}$ & No. of seed pod ${ }^{-1}$ & No. of seed plant ${ }^{-1}$ & 100 seed weight $(\mathrm{g})$ & Yield palnt $^{-1}$ \\
\hline LM-123-7 & $201 \mathrm{a}-\mathrm{c}$ & $1.57 \mathrm{a}-\mathrm{c}$ & $308 a-c$ & $2.63 \mathrm{ab}$ & $7.99 \mathrm{a}$ \\
\hline LM-132-7 & $179 a-c$ & $1.55 \mathrm{a}-\mathrm{c}$ & $301 \mathrm{a}-\mathrm{c}$ & 2.46 a-d & $7.68 \mathrm{ab}$ \\
\hline LM-28-2 & $121 \mathrm{bc}$ & $1.58 \mathrm{a}-\mathrm{c}$ & $188 \mathrm{bc}$ & $2.09 \mathrm{c}-\mathrm{g}$ & $4.03 \mathrm{a}-\mathrm{c}$ \\
\hline LM-14-2 & $152 \mathrm{bc}$ & $1.37 \mathrm{a}-\mathrm{d}$ & $211 \mathrm{bc}$ & $2.55 a-c$ & $5.52 \mathrm{a}-\mathrm{c}$ \\
\hline LM-21-6 & $154 \mathrm{bc}$ & $1.42 \mathrm{a}-\mathrm{d}$ & $221 \mathrm{a}-\mathrm{c}$ & $2.37 \mathrm{~b}-\mathrm{d}$ & $5.39 a-c$ \\
\hline LM-24-3 & $155 \mathrm{bc}$ & $1.54 \mathrm{a}-\mathrm{c}$ & $239 a-c$ & $2.85 \mathrm{a}$ & $6.90 \mathrm{a}-\mathrm{c}$ \\
\hline LM-37-8 & $122 \mathrm{bc}$ & $1.61 \mathrm{a}-\mathrm{c}$ & $199 \mathrm{bc}$ & $1.54 \mathrm{~h}$ & $3.16 \mathrm{bc}$ \\
\hline LM-48-1 & $124 \mathrm{bc}$ & $1.58 \mathrm{ac}$ & $193 \mathrm{bc}$ & $2.27 \mathrm{~b}-\mathrm{e}$ & $4.03 \mathrm{a}-\mathrm{c}$ \\
\hline LM-99-4 & $154 \mathrm{bc}$ & $1.54 \mathrm{a}-\mathrm{c}$ & $249 a-c$ & $2.44 \mathrm{a}-\mathrm{d}$ & $6.11 \mathrm{a}-\mathrm{c}$ \\
\hline LM-95-3 & $149 \mathrm{bc}$ & $1.41 \mathrm{a}-\mathrm{d}$ & $190 \mathrm{bc}$ & $2.41 \mathrm{a}-\mathrm{d}$ & $4.65 a-c$ \\
\hline LM-13-1 & $192 a-c$ & $1.68 \mathrm{a}$ & $322 a-c$ & $2.24 b-f$ & $7.27 \mathrm{ab}$ \\
\hline LM-156-1 & $175 a-c$ & $1.51 \mathrm{a}-\mathrm{d}$ & $261 a-c$ & $1.78 \mathrm{~b}-\mathrm{h}$ & $4.88 \mathrm{a}-\mathrm{c}$ \\
\hline LM-185-2 & $158 \mathrm{bc}$ & $1.49 \mathrm{a}-\mathrm{d}$ & $247 a-c$ & $2.17 b-f$ & $5.56 \mathrm{a}-\mathrm{c}$ \\
\hline LM-20-3 & $142 \mathrm{bc}$ & $1.35 b-d$ & 204 bc & $1.79 \mathrm{f}-\mathrm{h}$ & $3.50 \mathrm{a}-\mathrm{c}$ \\
\hline LM-67-5 & $166 \mathrm{a}-\mathrm{c}$ & $1.31 \mathrm{~cd}$ & $221 a-c$ & $2.30 \mathrm{~b}-\mathrm{e}$ & $5.18 \mathrm{a}-\mathrm{c}$ \\
\hline LM-75-4 & $116 \mathrm{bc}$ & $1.55 \mathrm{a}-\mathrm{c}$ & $194 \mathrm{bc}$ & $2.01 \mathrm{~d}-\mathrm{g}$ & $4.28 \mathrm{a}-\mathrm{c}$ \\
\hline ICARDA 2348 & $288 a$ & $1.46 \mathrm{a}-\mathrm{d}$ & $418 a$ & $1.85 \mathrm{e}-\mathrm{h}$ & $7.65 a b$ \\
\hline ICARDA 38211 & $126 \mathrm{bc}$ & $1.36 \mathrm{a}-\mathrm{d}$ & $170 \mathrm{c}$ & $2.23 b-f$ & $3.84 a-c$ \\
\hline ICARDA 23136 & $120 \mathrm{bc}$ & $1.21 \mathrm{~d}$ & $149 \mathrm{c}$ & $1.68 \mathrm{gh}$ & $2.52 \mathrm{c}$ \\
\hline ICARDA 23121 & $105 c$ & $1.63 \mathrm{ab}$ & $173 \mathrm{c}$ & $2.01 \mathrm{~d}-\mathrm{g}$ & $3.24 \mathrm{bc}$ \\
\hline ICARDA 23105 & $119 \mathrm{bc}$ & $1.58 \mathrm{a}-\mathrm{c}$ & $181 \mathrm{bc}$ & $2.17 b c-f$ & $4.00 \mathrm{a}-\mathrm{c}$ \\
\hline ICARDA 23128 & $241 \mathrm{ab}$ & $1.55 \mathrm{a}-\mathrm{c}$ & $384 a b$ & $1.68 \mathrm{gh}$ & $6.13 a-c$ \\
\hline CV (\%) & 14.82 & 15.90 & 5.77 & 6.95 & 8.25 \\
\hline Maximum & 288 & 1.68 & 418 & 2.85 & 7.99 \\
\hline Minimum & 104 & 1.21 & 149 & 1.54 & 2.52 \\
\hline Mean & 157.17 & 1.49 & 237.44 & 2.16 & 5.16 \\
\hline LSD (0.05) & 106.30 & 0.26 & 170.10 & 0.40 & 3.78 \\
\hline
\end{tabular}


Table 3. Heritability $\left(h^{2} b\right)$ of different traits of 22 lentil genotypes

\begin{tabular}{|l|c|}
\hline \multicolumn{1}{|c|}{ Traits } & Heritability, $\mathrm{h}^{2} \mathrm{~b}(\%)$ \\
\hline Plant height & 73.40 \\
\hline Days to 50\% flowering & 91.67 \\
\hline Days to maturity & 82.65 \\
\hline Number of primary branches plant $^{-1}$ & 70.40 \\
\hline Number of secondary branches plant $^{-1}$ & 62.76 \\
\hline Height of the lowest pod $^{-1}$ & 77.79 \\
\hline Number of pod plant $^{-1}$ & 57.43 \\
\hline Number of seeds pod $^{-1}$ & 62.32 \\
\hline Number of seeds plant $^{-1}$ & 57.40 \\
\hline 100- Seed weight & 85.12 \\
\hline Yield plant $^{-1}$ & 58.93 \\
\hline
\end{tabular}

Relationship between physiological and yield contributing characters was studied through analysis of correlation between them. Grain yield plant ${ }^{-1}$ was positively correlated with pod plant ${ }^{-1}$, seed pod ${ }^{-1}$, seed plant $^{-1}$ and 100 seed weight. Among them 100 seed weight was significantly correlated with grain yield plant $^{-1}$ suggesting that genotypes with high partitioning efficiency gave increase in grain yield plant ${ }^{-1}$ (Table 4). The path coefficient analysis (Table 5) was performed using correlation coefficient to determine direct and indirect influence considering eight characters viz. plant height, days to flowering, days to maturity, number of primary branches plant ${ }^{-1}$, number of secondary branches plant $^{-1}$, number of pods plant $^{-1}$, number of seeds pod $^{-1}$ and 100-seed weight to yield plant ${ }^{-1}$. Days to maturity, number of primary branches plant ${ }^{-1}$, number of secondary branches plant ${ }^{-1}$, pods plant ${ }^{-1}$, seeds pod $^{-1}$ and 100 -seed weight had positive direct effects on grain yield plant ${ }^{-1}$ which is agreement with the findings of Rasheed et al. (2008) and Younis et al. (2008). Among the characters pods plant ${ }^{-1}$ and 100-seed weight had high positive direct effects on grain yield plant ${ }^{-1}$. Plant height, and days to flowering showed negative direct effects on grain yield plant ${ }^{-1}$. The highest direct effect of number of pod plant ${ }^{-1}$ gave a significant positive correlation inducing that among all the traits under study these trait contributed maximum for grain yield. The residual effect was 0.27 indicating that the eight characters contributed 73 percent of variability in grain yield plant ${ }^{-1}$ studied in path analysis. The present study suggested that plant height, days to flowering, days to maturity, number of primary branches plant ${ }^{-1}$, number of secondary branches plant ${ }^{-1}$, pods plant ${ }^{-1}$, and seeds pod $^{-1}, 100$-seed weight should be included owing to importance in selecting the genotypes for higher grain yield in lentil. Based on $D^{2}$-value, the genotypes were grouped into five clusters viz. I, II, III, IV and V (Table 6).

Table 4. Coefficients of correlation among different yield components of 22 lentil genotypes

\begin{tabular}{|c|c|c|c|c|c|c|c|c|c|c|}
\hline Traits & $\begin{array}{l}\text { Plant } \\
\text { height } \\
(\mathrm{cm})\end{array}$ & $\begin{array}{c}\text { Days to } \\
50 \% \\
\text { flowering }\end{array}$ & $\begin{array}{l}\text { Days of } \\
\text { maturity }\end{array}$ & $\begin{array}{c}\text { Number of } \\
\text { primary } \\
\text { branches } \\
\text { plant }{ }^{-1} \\
\end{array}$ & $\begin{array}{c}\text { Number of } \\
\text { secondary } \\
\text { branches } \\
\text { plant }^{-1}\end{array}$ & $\begin{array}{l}\text { Height of } \\
\text { the lowest } \\
\text { pod }(\mathrm{cm})\end{array}$ & $\begin{array}{l}\text { Number } \\
\text { of pod } \\
\text { plant }{ }^{-1}\end{array}$ & $\begin{array}{l}\text { Number } \\
\text { of seeds } \\
\text { pod-1 }^{-1}\end{array}$ & $\begin{array}{c}\text { Number } \\
\text { of seeds } \\
\text { plant }^{-1}\end{array}$ & $\begin{array}{l}100-\text { Seed } \\
\text { weight }(\mathrm{g})\end{array}$ \\
\hline Days to $50 \%$ flowering & 0.388 & & & & & & & & & \\
\hline Days of maturity & 0.308 & $0.580^{\star *}$ & & & & & & & & \\
\hline Number of primary branches plant:-1 & 0.326 & $0.590^{\star *}$ & $0.454^{*}$ & & & & & & & \\
\hline Number of secondary branches plant ${ }^{-1}$ & $0.532^{\star}$ & $0.672^{\star \star}$ & 0.402 & $0.769 * \star$ & & & & & & \\
\hline Height of the lowest pod $(\mathrm{cm})$ & $0.612^{\star \star}$ & $0.551^{\star \star}$ & 0.371 & 0.359 & $0.497^{*}$ & & & & & \\
\hline Number of pods plant ${ }^{-1}$ & 0.219 & 0.196 & 0.098 & 0.079 & 0.267 & -0.127 & & & & \\
\hline Number of seeds pod ${ }^{-1}$ & 0.018 & -0.207 & -0.326 & -0.412 & -0.077 & -0.149 & 0.054 & & & \\
\hline Number of seeds plant-1 & 0.23 & 0.13 & 0.033 & 0.011 & 0.267 & -0.187 & $0.960^{\star \star}$ & 0.291 & & \\
\hline 100- Seed weight $(\mathrm{g})$ & $-0.480^{*}$ & $-0.580^{\star \star}$ & -0.263 & -0.413 & -0.404 & -0.257 & -0.026 & 0.071 & -0.024 & \\
\hline Yield plant ${ }^{-1}(\mathrm{~g})$ & -0.109 & -0.290 & -0.129 & -0.227 & -0.037 & -0.35 & $0.772^{\star \star}$ & 0.289 & $0.820^{\star \star}$ & $0.524^{*}$ \\
\hline
\end{tabular}

* and ** indicates significant at 0.05 and 0.01 probability, respectively 
Table 5. Partitioning of phenotypic correlations into direct and indirect effects of eight important characters by path analysis

\begin{tabular}{|c|c|c|c|c|c|c|c|c|c|}
\hline Characters & $\begin{array}{l}\text { Plant } \\
\text { height }\end{array}$ & $\begin{array}{c}\text { Days to } \\
50 \% \\
\text { flowering }\end{array}$ & $\begin{array}{l}\text { Days of } \\
\text { maturity }\end{array}$ & $\begin{array}{c}\text { Number of } \\
\text { primary } \\
\text { branches } \\
\text { plant-1 }\end{array}$ & $\begin{array}{c}\text { Number of } \\
\text { secondary } \\
\text { branches } \\
\text { plant-1 }\end{array}$ & $\begin{array}{l}\text { Number } \\
\text { of pod } \\
\text { plant }^{-1}\end{array}$ & $\begin{array}{l}\text { Number of } \\
\text { seeds pod }-1\end{array}$ & $\begin{array}{c}\text { 100-Seed } \\
\text { weight }\end{array}$ & $\begin{array}{l}\text { Correlation to } \\
\text { yield plant-1 }\end{array}$ \\
\hline Plant height & -0.070 & -0.027 & -0.022 & -0.023 & -0.037 & -0.015 & -0.001 & 0.033 & -0.109 \\
\hline Days to $50 \%$ flowering & -0.104 & -0.267 & -0.155 & -0.158 & -0.179 & -0.053 & 0.056 & 0.155 & -0.290 \\
\hline Days of maturity & 0.031 & 0.057 & 0.099 & 0.044 & 0.040 & 0.010 & -0.031 & -0.026 & -0.129 \\
\hline Number of primary branches plant ${ }^{-1}$ & 2.182 & 0.039 & 0.030 & 0.066 & 0.051 & 0.005 & -0.027 & -0.027 & -0.227 \\
\hline Number of secondary branches plant ${ }^{-1}$ & 0.027 & 0.034 & 0.020 & 0.039 & 0.050 & 0.014 & -0.004 & -0.020 & -0.037 \\
\hline Number of pod plant ${ }^{-1}$ & 0.178 & 0.162 & 0.081 & 0.065 & 0.219 & 0.811 & 0.041 & 0.024 & $0.772^{\star \star}$ \\
\hline Number of seeds pod-1 & 0.005 & -0.048 & -.076 & -0.094 & -0.018 & 0.011 & 0.230 & 0.016 & 0.289 \\
\hline 100- Seed weight & -0.198 & -.239 & -.107 & -0.169 & -0.165 & -0.012 & 0.029 & 0.413 & $0.524^{*}$ \\
\hline Residual effect $=0.27$ & & & & & & & & & \\
\hline
\end{tabular}

${ }^{*}$ and ${ }^{* *}$ indicates significant at 0.05 and 0.01 probability, respectively.

Bold figures indicate the direct effect

Table 6. Clustering pattern of 22 genotypes of based on Euclidean distance following Ward's method and the member present in each respective cluster

\begin{tabular}{|c|c|c|c|}
\hline Cluster number & Number of genotypes & Percent & Name of genotypes \\
\hline I & 4 & 18.18 & LM-123-7, LM-132-7, LM-24-3 and LM-13-1 \\
\hline II & 8 & 36.36 & $\begin{array}{c}\text { LM-28-2, LM-14-2, LM-21-6, LM-37-8, LM-48- } \\
\text { 1, LM-99-4, LM-95-3 and LM-75-4 }\end{array}$ \\
\hline III & 4 & 18.18 & LM-156-1, LM-185-2, LM-20-3 and LM-67-5 \\
\hline IV & 2 & 9.09 & ICARDA 2348 and ICARDA 23128 \\
\hline V & 4 & 18.18 & ICARDA 38211, ICARDA 23136, ICARDA \\
& & & 23121 and ICARDA 23105 \\
\hline
\end{tabular}

Table 7. Average intra and inter-cluster $D^{2}$ and $D$ values of five clusters

\begin{tabular}{|c|c|c|c|c|c|}
\hline Cluster & I & II & III & IV & V \\
\hline I & $\frac{16953}{(130)}$ & $\begin{array}{c}53104 \\
(230)\end{array}$ & $\begin{array}{c}19199 \\
(139)\end{array}$ & $\begin{array}{c}167623 \\
(409)\end{array}$ & $\begin{array}{c}106170 \\
(326)\end{array}$ \\
\hline II & & $\frac{\mathbf{1 2 7 8 9}}{(113)}$ & $\begin{array}{l}18151 \\
(135)\end{array}$ & $\begin{array}{c}373299 \\
(611)\end{array}$ & $\begin{array}{c}19249 \\
(139)\end{array}$ \\
\hline III & & & $\frac{\mathbf{1 2 7 0 8}}{(113)}$ & $\begin{array}{c}249447 \\
(499)\end{array}$ & $\begin{array}{l}51891 \\
(228)\end{array}$ \\
\hline IV & & & & $\frac{48091}{(219)}$ & $\begin{array}{c}496167 \\
(704)\end{array}$ \\
\hline $\mathrm{V}$ & & & & & $\frac{10621}{(103)}$ \\
\hline
\end{tabular}

Cluster I, III and V had same number of genotypes i.e. four and cluster II contained eight genotypes and cluster IV contained only two genotypes. The highest intra cluster distance was found in cluster IV. The maximum inter cluster distance was observed between genotypes of cluster IV and $V$ followed by clusters II and IV (Table 7 and Fig.1). Thus, hybridization among genotypes drawn from these widely divergent clusters with high yield potential would likely to produce heterotic combinations and wide variability in segregating generations. 


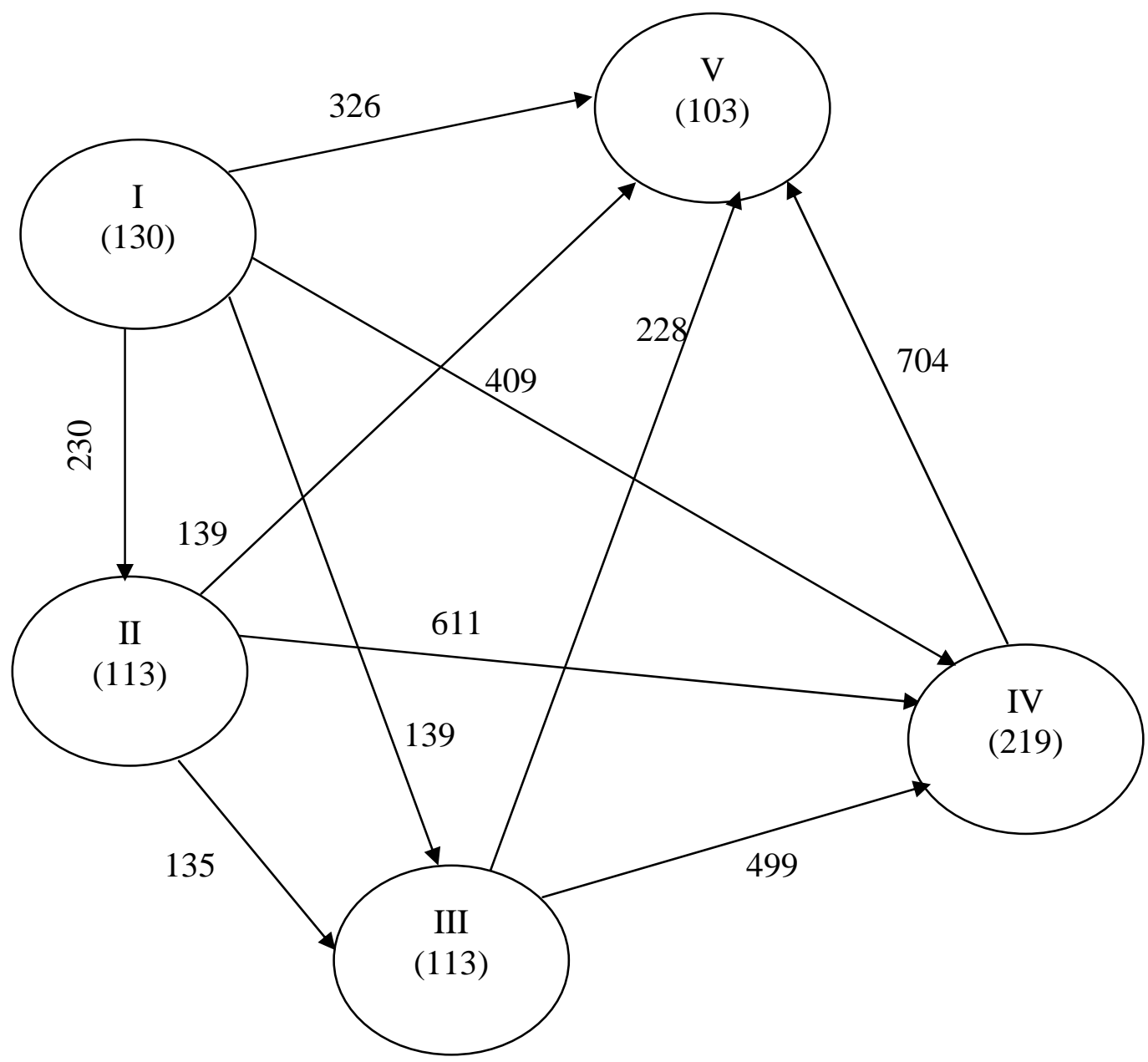

Fig. 1. Cluster diagram showing the average intra and inter cluster distances $\left(D=\sqrt{D^{2}}\right.$ values) of 22 lentil genotypes. The values along the lines inter cluster distances and the values within the circle indicate intra cluster distances

Dendrogram based on Ward's method indicated grouping of 22 genotypes of lentil into five clusters (Fig. 2). LM-123-7, LM-132-7, LM-13-1 and LM-24-3 were grouped in cluster I with high genetic (130) distance, while LM-28-2, LM-14-2, LM-21-6, LM-37-8, LM-48-1, LM-99-4, LM-95-3 and LM-75-4 in cluster II with 113 and LM-156-1, LM-185-2, LM-20-3 and LM-67-5 in cluster III with 113. ICARDA 2348 and ICARDA 23128 were grouped in cluster IV with highest genetic (219) distance. ICARDA 38211, ICARDA 23136, ICARDA 23121 and ICARDA 23105 were grouped in cluster V with lowest genetic (103) distance. 
Dendrogram

Ward's Method, Euclidean
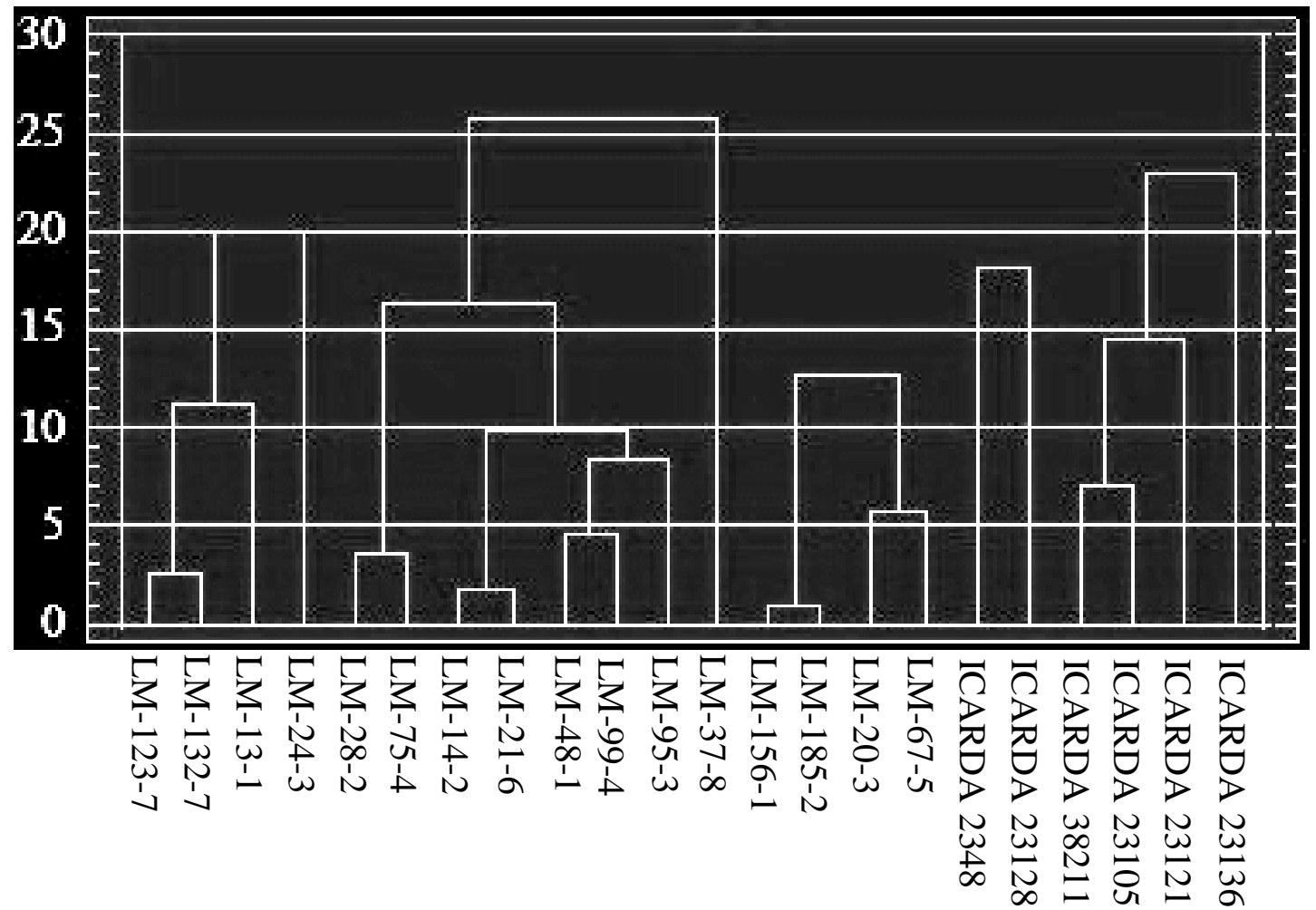

Fig. 2. Dendrogram based on summarized data on differentiation among 22 genotypes of lentil according to Ward's method

The mean values of each cluster for eleven characters are presented in Table 8. There was wide range of variation in the cluster mean values for all the characters. The mean values of all characters for the respective character were categorized into low $(\mathrm{L})$, intermediate $(\mathrm{I})$ and high $(\mathrm{H})$ classes. Genotypes belong to cluster I showed high number of seed $\operatorname{pod}^{-1}$, maximum 100 -seed weight $(\mathrm{g})$ and yield plant ${ }^{-1}(\mathrm{~g})$ then the rest of the clusters. Maximum days to maturity was found in cluster III then other cluster and minimum days to maturity was found in cluster I. Genotypes belong to cluster IV showed higher plant height $(\mathrm{cm})$, days to flowering, number of secondary branches plant ${ }^{-1}$, number of pod plant ${ }^{-1}$ and number of seed plant ${ }^{-1}$ then the other cluster. Genotypes belong to cluster V showed higher number of primary branches plant ${ }^{-1}$ and height of the lowest pod.

Table 8. Cluster mean for 11 yield and yield related characters in 22 genotypes

\begin{tabular}{|c|c|c|c|c|c|}
\hline Cluster & $\mathrm{I}$ & $\mathrm{II}$ & $\mathrm{III}$ & $\mathrm{IV}$ & $\mathrm{V}$ \\
\hline Plant height $(\mathrm{cm})^{\text {Days to flowering }}$ & $28.12(\mathrm{I})$ & $27.44(\mathrm{~L})$ & $28.80(\mathrm{I})$ & $34.70(\mathrm{H})$ & $32.05(\mathrm{I})$ \\
\hline Days to maturity & $51.95(\mathrm{~L})$ & $56.00(\mathrm{I})$ & $59.20(\mathrm{I})$ & $68.70(\mathrm{H})$ & $64.65(\mathrm{I})$ \\
\hline No. of primary branches plant $^{-1}$ & $104.55(\mathrm{~L})$ & $105.27(\mathrm{I})$ & $109.20(\mathrm{H})$ & $108.50(\mathrm{I})$ & $108.75(\mathrm{I})$ \\
\hline No. of secondary branches plant $^{-1}$ & $11.85(\mathrm{I})$ & $3.80(\mathrm{~L})$ & $5.40(\mathrm{I})$ & $5.50(\mathrm{I})$ & $6.05(\mathrm{H})$ \\
\hline Height of the lowest pod $_{\text {Number of pod plant }}^{-1}$ & $6.17(\mathrm{~L})$ & $9.53(\mathrm{~L})$ & $12.00(\mathrm{I})$ & $15.70(\mathrm{H})$ & $14.90(\mathrm{I})$ \\
\hline Number of seed pod $^{-1}$ & $181.55(\mathrm{I})$ & $136.40(\mathrm{I})$ & $160.30(\mathrm{I})$ & $264.70(\mathrm{H})$ & $117.45(\mathrm{~L})$ \\
\hline Number of seed plant $^{-1}$ & $1.58(\mathrm{H})$ & $1.51(\mathrm{I})$ & $1.41(\mathrm{~L})$ & $1.51(\mathrm{I})$ & $1.45(\mathrm{I})$ \\
\hline 100 seed weight $(\mathrm{g})^{\text {Yield plant }^{-1}(\mathrm{~g})}$ & $292.60(\mathrm{I})$ & $205.68(\mathrm{I})$ & $233.25(\mathrm{I})$ & $401.30(\mathrm{H})$ & $168.05(\mathrm{~L})$ \\
\hline & $2.55(\mathrm{H})$ & $2.21(\mathrm{I})$ & $2.01(\mathrm{I})$ & $1.77(\mathrm{~L})$ & $2.02(\mathrm{I})$ \\
\hline
\end{tabular}

$\mathrm{H}=$ High value, $\mathrm{I}=$ Intermediate value, and $\mathrm{L}=\mathrm{Low}$ value 
Table 9. Selection score, rank and expected genetic gain of 21 genotypes of lentil considering nine characters

\begin{tabular}{|c|c|c|c|c|}
\hline SI. No. & Genotypes & Selection score & Rank & Expected genetic gain \\
\hline 1 & LM-123-7 & 7636 & 1 & \multirow{21}{*}{ - } \\
\hline 2 & LM-132-7 & 6813 & 2 & \\
\hline 3 & LM-28-2 & 4716 & 7 & \\
\hline 4 & LM-14-2 & 5828 & 5 & \\
\hline 5 & LM-21-6 & 5952 & 3 & \\
\hline 6 & LM-24-3 & 5869 & 4 & \\
\hline 7 & LM-37-8 & 4759 & 6 & \\
\hline 8 & LM-48-1 & 4228 & 8 & \\
\hline 9 & LM-99-4 & -167 & 10 & \\
\hline 10 & LM-95-3 & -231 & 14 & \\
\hline 11 & LM-13-1 & -252 & 15 & \\
\hline 12 & LM-156-1 & -209 & 11 & \\
\hline 13 & LM-185-2 & -286 & 16 & \\
\hline 14 & LM-20-3 & -367 & 17 & \\
\hline 15 & LM-67-5 & -223 & 13 & \\
\hline 16 & LM-75-4 & -383 & 18 & \\
\hline 17 & ICARDA 2348 & -215 & 12 & \\
\hline 18 & ICARDA 38211 & 401 & 9 & \\
\hline 19 & ICARDA 23121 & -497 & 19 & \\
\hline 20 & ICARDA 23105 & -577 & 21 & \\
\hline 21 & ICARDA 23128 & -571 & 20 & \\
\hline
\end{tabular}

Among 22 genotypes of lentil, LM-123-7 possessed the highest selection score index (7636) and rank as the best followed by LM-132-7, LM-21-6, LM-24-3 and LM-14-2 with 6813, 5952, 5869 and 5828 respectively (Table 9). The genotype ICARDA 23105 was worst having the lowest selection score of -577 followed by ICARDA 23128 (-571) and ICARDA 23121 (-497). The expected genetic gain $(\Delta G)$ was 143 at $5 \%$ selection intensity i.e. 2-3 highest scoring genotypes from these 21 lentil genotypes might be recommended for farmers' cultivation for better yield.

\section{References}

Dutta, R.K. 2001. Assessment of the advanced mutant lines/varieties of BINA with emphasis on physiological criteria. Report on ARMP Project No. 512, BARC, Farmgate, Dhaka-1215.

FAOSTAT. 2010. (faostat.fao.org/site/567/DesktopDefault.aspx?PagelD=567\#ancor, visited on 08.05.2012).

Ferguson, M. and Erskine, W. 2001. Lentils (Lens culinaris L.). In: Maxted, N. and S. J. Bennett., Plant Genetic Resources of Legumes in the Mediterranean. Kluwer Academic Publishers, Dordrecht, Netherlands, pp. 132-157.

Ford, R., Rubeena, Redden, R., Materne, M. and Taylor, P. 2007. Genome mapping and molecular breeding in lentil: Lentil In: enome Mapping and Molecular Breeding. Volume III, Pulse, Sugar and Starch Crops, Ed. C. Kole. Springer (Heidelberg, Berlin, New York, Tokyo). pp. 91-108.

Rasheed, S., Hanif, M., Sadiq, S., Abbas, G., Asghar, M.J. and Haq, M.A. 2008. Inheritance of seed yield and related traits in some lentil (Lens culinaris Medik) genotypes. Pakistan. J. Agril. Sci., 45(3): 49-52.

Sarker, A., Erskine, W. and Singh, M. 2005. Variation in shoot and root characteristics and their association with drought tolerance in lentil landraces. Genet. Resour. Crop Evol., 52(1): 89-97.

Utz, H.F. 2007. PLABSTAT (Version 2N), A computer program for the computation of variances and covariances. Institute of Plant Breeding, Seed Science, and Population Genetics, University of Hohenheim, Stuttgart, Germany (http://www.unihohenheim.de/ipspwww/soft.html cited 25 August 2007).

Younis, N., Hanif, M., Sadiq, S., Abbas, G., Asghar, M.J. and Haq, M.A. 2008. Estimation of Genetic Parameters and Path Analysis in Lentil. Pakistan J. Agr. Sci. 45(3):44-48.

Zohary, D. 1972. The wild progenitor and place of origin of the cultivated lentil Lens culinaris. Econ. Bot., 26: 326-332. 